\title{
Cardiopulmonary Exercise Testing in Coronary Artery Disease Male Aviators Prior to Refighting: A Suggested Modality
}

\author{
Ashish Chauhan ${ }^{1}$, Manvinder Pal Singh Marwaha², Vipin Sharma ${ }^{3}$, Rajesh Vaidya ${ }^{4}$, Bhupinder Kaur Anand ${ }^{5}$ \\ ${ }^{1}$ Senior Advisor Medicine \& Cardiologist, Air Fource Central Medical Establishment, Subroto Park, New Delhi, ${ }^{2}$ Senior Advisor Aerospace Medicine, Air Force \\ Central Medical Establishment, Subroto Park, New Delhi, ${ }^{3}$ Senior Advisor Aerospace Medicine, Institute Of Aerospace Medicine, Bengalru, Karnataka, ${ }^{4}$ Air Officer \\ Commanding, Air Force Central Medical Establishment, Subroto Park, New Delhi, ${ }^{5}$ Professor, Department Of Community Medicine, SGT Medical College \\ Gurugram.
}

\section{Abstract}

Background: Exercise testing provides valuable diagnostic and prognostic information regarding patients with cardiovascular and pulmonary disease. The aim of this study is to assess maximum oxygen consumption (O2 Max), oxygen consumption at Lactate threshold and exercise ECG, heart rate, BP response in aviators with CAD who are being considered for refighting. Subjects and Methods: We included 7 males from age group 35 - 56 y who were CAD patients for medical evaluation at our center. A normogram was earlier obtained by subjecting 72 males between the age $18-60$ year by random selection during medical for initial entry to Airforce and routine annual medical in serving personnel. Results: These males were subjected to cardiopulmonary stress test, after an echocardiogram was done to assess structural heart disease. The data obtained was compared with the normogram for age. Conclusion: In this study, we conclude that in our population a healthy aviator reaches an average of $80 \%$ of age predicted VO2 max (normogram for age 30 - 39 and 40 - 49 attached above). Aviator no, 2,3,4,5,7 were reflighted,

Keywords: Cardiopulmonary exercise, Coronary Artery Disease, Myocardial infarction.

Corresponding Author: Dr. Manvinder Pal Singh Marwaha, Senior Advisor Aerospace Medicine, Air Force Central Medical Establishment, Subroto Park, New Delhi.

Received: July 2019

Accepted: July 2019

\section{Introduction}

In several Air Forces around the world an aviator is not permitted to fly after a myocardial infarction as he has a definite risk of developing an arrhythmia from the scar region (Scar VT/ VF); there are apprehensions about performance abilities and in flight recurrence of a MI. In the Indian Air Force, refighting is permitted in transport aircraft provided the pilot has normal echocardiogram, Holter, a negative TMT and CAG does not reveal significant disease in epicardial arteries other than infarct related artery.

Exercise testing provides valuable diagnostic and prognostic information regarding patients with cardiovascular and pulmonary disease. The addition of ventilatory gas exchange measurements during exercise testing allow for the analysis of gas exchange at rest, during exercise, and during recovery and yield measures of oxygen uptake $(\mathrm{O} 2)$, carbon dioxide output (CO2), and ventilation (E). These data can be readily integrated with standard variables measured during exercise testing, including heart rate, blood pressure, work rate, electrocardiography findings, and symptoms, to provide a comprehensive assessment of exercise tolerance and exercise responses. ${ }^{[1]}$ CAD patients are often smokers and may have associated
COPD which affects their exercise capacity. Maximum oxygen consumption (O2 Max) is a physiological measure of combined efficacy of heart - lungs - muscles and metabolic combine. Cardiopulmonary exercise testing (CPET) provides assessment of the integrative exercise responses involving the pulmonary, cardiovascular, haematopoietic, neuropsychological, and skeletal muscle systems, which are not adequately reflected through the measurement of individual organ system function. This non- invasive, dynamic physiological overview permits the evaluation of both submaximal and peak exercise responses, providing the doctor with relevant information for clinical decision making. CPET is increasingly being used in a wide spectrum of clinical applications for the evaluation of undiagnosed exercise intolerance and for the objective determination of functional capacity and impairment. Its use in patient management is increasing with the understanding that resting pulmonary and cardiac function testing cannot reliably predict exercise performance and functional capacity and that overall health status correlates better with exercise tolerance than with resting measurements. ${ }^{[1]}$ To assess maximum oxygen consumption (O2 Max), oxygen consumption at Lactate threshold and exercise ECG, heart rate, $\mathrm{BP}$ response in aviators with $\mathrm{CAD}$ who are being considered for refighting. 


\section{Subjects and Methods}

\section{Study Population}

We included 7 males from age group $35-56$ y who were CAD patients for medical evaluation at our centre. A normogram was earlier obtained by subjecting 72 males between the age $18-60$ year by random selection during medical for initial entry to Airforce and routine annual medical in serving personnel.

\section{Study Area}

This study was conducted in the department of cardiology in the Air Fource Central Medical Establishment, Subroto Park, New Delhi.

\section{Study Duration}

The duration of the study was over a period of from 2011 to 2013.

\section{Data Collection}

CAD patients were subjected to CPET (Cardio Pulmonary Exercise Test) after assessing structural heart disease by echocardiogram. QUARK CPET coupled along with Mortara treadmill was used. Bruce protocol for graded exercise was used to standardize results of stress test along with ventilatory protocols.

Quark CPET is manufactured by Cosmed and gives $01 \mathrm{~min}$ analysis of oxygen consumption, $\mathrm{CO} 2$ production. It has an inflow and an outflow sensor for oxygen and $\mathrm{CO} 2$, which enables these measurements. The lactate threshold is a derived parameter from this. There is a turbine in the breathing apparatus which measures flow rates and minute ventilation. The equipment needs preparation with regular calibration using a standard premixed gas cylinder supplied by the company. The individual is made to wear the breathing mask in an airtight manner and then made to exercise on the treadmill [Figure 1,2]

\section{Data Analysis}

Data were analyzed by using Microsoft excel.

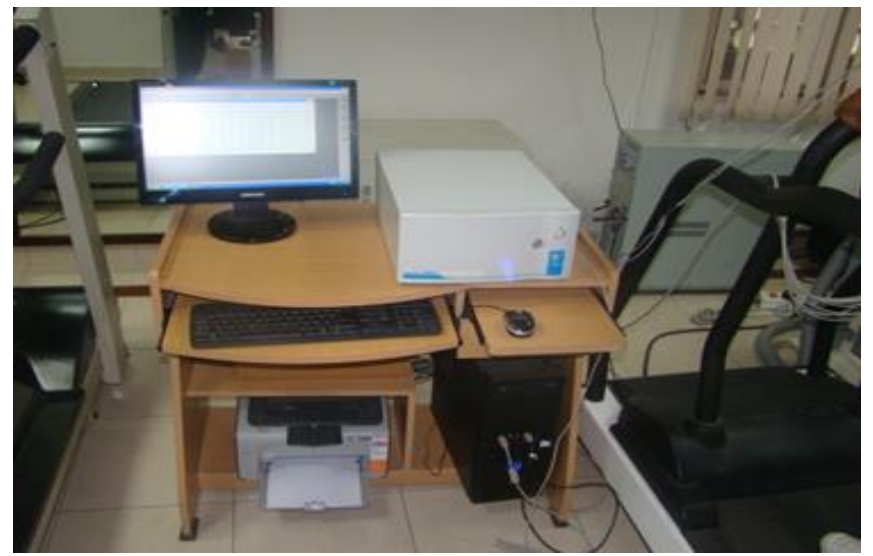

Figure 1:

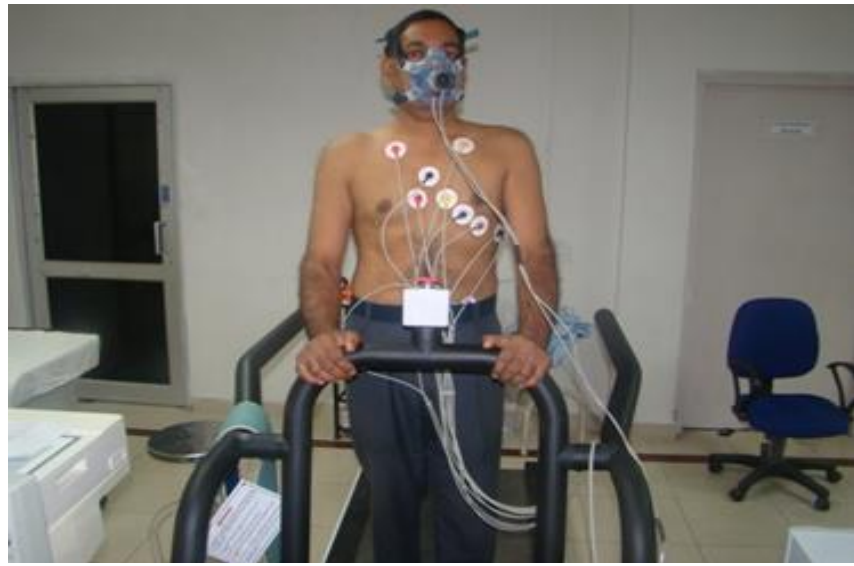

Figure 2:

\section{Results}

Table 1: Normogram (own data) Age 30 - 39 y

\begin{tabular}{|l|l|l|l|l|}
\hline & & VO2 MAX (mI/Kg/min) & \% Pred. & METS \\
\hline Smoker & 1 & 22.62 & 48 & 6.4 \\
\hline & 2 & 41.33 & 76 & 11.8 \\
\hline & 3 & 39.48 & 81 & 11.2 \\
\hline & 4 & 35.64 & 76 & 10.1 \\
\hline & 5 & 34.88 & 75 & 9.9 \\
\hline & 6 & 39.36 & & 11.2 \\
\hline & 7 & 38.81 & 75 & 8.2 \\
\hline & 8 & 35.8 & 84 & 10.2 \\
\hline & 9 & 32.16 & 72 & 9.1 \\
\hline & 10 & 28.56 & 64 & 8.1 \\
\hline & 11 & 32.5 & 70 & 9.2 \\
\hline Smoker & 12 & 24.15 & 54 & 6.9 \\
\hline & 13 & 28.5 & 77 & 8.1 \\
\hline & 14 & 26.46 & 58 & 7.5 \\
\hline Smoker & 15 & 34.45 & 76 & 9.8 \\
\hline Smoker & 16 & 34.55 & 68 & 9.8 \\
\hline & 17 & 31.33 & 72 & 8.9 \\
\hline
\end{tabular}

Table 2: Normogram (own data) Age 40 - 49 y

\begin{tabular}{|l|l|l|l|l|}
\hline & VO2 MAX (ml/Kg/min) & \% Pred. & METS & \\
\hline 1 & 37.86 & 93 & 10.8 & \\
\hline 2 & 28.3 & 78 & 8 & Smoker \\
\hline 3 & 35.31 & 87 & 10 & \\
\hline 4 & 30.01 & 78 & 8.5 & \\
\hline 5 & 30.13 & 76 & 8.6 & \\
\hline 6 & 25.43 & 72 & 7.2 & \\
\hline 7 & 29.65 & 77 & 8.4 & \\
\hline 8 & 38.75 & 105 & 11 & \\
\hline 9 & 28.73 & 85 & 8.2 & \\
\hline 10 & 31.92 & 81 & 9.1 & \\
\hline 11 & 32.48 & 80 & 9.2 & \\
\hline 12 & 32.78 & 78 & 9.3 & \\
\hline 13 & 35.03 & 88 & 10 & \\
\hline 14 & 42.83 & 110 & 12.2 & \\
\hline 15 & 42.7 & 99 & 12.2 & \\
\hline 16 & 29.51 & 72 & 8.4 & Smoker \\
\hline 17 & 27.91 & 65 & 7.9 & \\
\hline 18 & 27.33 & 73 & 7.8 & \\
\hline 19 & 31.03 & 75 & 8.8 & \\
\hline 20 & 29.13 & 69 & 8.3 & \\
\hline 21 & 33.37 & 95 & 9.5 & \\
\hline 22 & 34.64 & 82 & 9.8 & \\
\hline 23 & 27.69 & 65 & 7.9 & \\
\hline
\end{tabular}


These males were subjected to cardiopulmonary stress test, after an echocardiogram was done to assess structural heart disease. The data obtained was compared with the normogram for age. Selected parameters from the data obtained have been projected below:

\section{Table 3: CAD Patients}

\begin{tabular}{|l|l|l|l|l|l|l|l|l|l|}
\hline $\begin{array}{l}\text { S. } \\
\text { No. }\end{array}$ & Age & $\begin{array}{l}\text { Wt } \\
\text { Kg }\end{array}$ & $\begin{array}{l}\text { Ht } \\
\mathbf{C m}\end{array}$ & $\begin{array}{l}\text { BMI } \\
\text { Kg/sqm }\end{array}$ & Diagnosis & LVEF & VO2 MAX LT & $\begin{array}{l}\text { VO2 MAX. } \\
\text { PRED }\end{array}$ & $\begin{array}{l}\text { \% } \\
\text { PRED }\end{array}$ \\
\hline 1 & 40 & 73 & 181 & 22.2 & AWMI(Old) Post CABG & $30-35 \%$ & 2.01 & 25.45 \\
\hline 2 & 50 & 59 & 166 & 21.4 & IWMI & $60 \%$ & 25.74 & 37 \\
\hline 3 & 43 & 88 & 170 & 30.4 & Post PTCA \& Stenting to RI & $60 \%$ & 18.39 & 36.52 \\
\hline 4 & 35 & 72 & 176 & 23.2 & AWMI (Post PTCA to LAD and LCX) & $50 \%$ & 40.91 & 24.37 & 36.49 \\
\hline 5 & 56 & 83 & 181 & 25.3 & $\begin{array}{l}\text { AWMI (Post PTCA to LAD SR stenting } \\
\text { Done) }\end{array}$ & $60 \%$ & 26.07 & 87 \\
\hline 6 & 44 & 80 & 176 & 25.8 & AWMI & $40 \%$ & 25.82 & 33.77 & 23.56 \\
\hline 7 & 54 & 61 & 163 & 22.9 & $\begin{array}{l}\text { CAD,PTCA \&Stenting to LCX and OM1, } \\
\text { Rep. PTCA \& stenting to LCX }\end{array}$ & $60 \%$ & 39.26 & 63 & 37.11 \\
\hline
\end{tabular}

\section{Discussion}

In several Air Force around the world an aviator is not permitted to fly after a myocardial infarction as he has a definite risk of developing an arrhythmia from the scar region (Scar VT/ VF); there are apprehensions about performance abilities and in flight recurrence of a MI. In the Indian Air Force, reflighting is permitted in transport aircraft provided the pilot has normal echocardiogram, Holter, a negative TMT and CAG does not reveal significant disease in epicardial arteries other than infarct related artery and there is no In stent restenosis.

In order to assess the cardiovascular system more physiologically and to permit flying in certain fit aviators with good effort tolerance despite a small myocardial scar, CPET is being suggested as the primary modality to assess physiological fitness.

The cardiovascular and respiratory systems work together to provide delivery system (of O2) and removal (of CO2) from the tissues (2). This happens by

(a) External respiration:

(i) Pulmonary ventilation

(ii) Pulmonary diffusion, or the exchange of $\mathrm{O} 2$ and $\mathrm{CO} 2$ between the lungs and the blood

(b) Internal respiration:

(i) Transport of $\mathrm{O} 2$ and $\mathrm{CO} 2$ in the blood;

(ii) Capillary gas exchange or the exchange of $\mathrm{O} 2$ and $\mathrm{CO} 2$ between the capillary blood and the working muscle.

Increase in oxygen uptake by the working muscles is contributed most by an increase in cardiac output (heart ratexstroke volume), which may increase to up to 6 times that at rest. ${ }^{[3]}$ Increase in blood flow to the lungs occurs by increased cardiac output and pulmonary vessel dilatation. This is accompanied by a greater extraction of $\mathrm{O} 2$ from the blood, which results in a widening of the arteriovenous oxygen (a-O2) difference. ${ }^{[4]}$

Increase in minute ventilation (E) during exercise must be matched by an increase in blood flow; that is, cardiac output must increase to appropriately match ventilation so that necessary gas exchange can occur. ${ }^{[5]}$ Mismatch occurs when cardiac output fails to increase as in heart failure, LV dysfunction etc and ventilation fails to increase in pulmonary conditions like COPD, Bronchial asthma, ILD etc. ${ }^{[6,7]}$ This is seen as increase in VE/ CO2 slope.

This study is an observation study to collect data with respect to aviators with coronary artery disease, who are being reflighted. The data was compared with baseline values obtained by us by subjecting 72 healthy males to CPET in different age groups. It was observed that the exercise in CPET as measured by actual oxygen consumption was lower than that derived by the duration of exercise done on only exercise / treadmill equipment (which is not coupled with ventilation parameters and derives METS by stages and duration of exercise). On an average, smokers had a lower predicted for age maximum oxygen consumption. The exercise training can be used to improve effort tolerance, delaying Lactate threshold and improve efficiency of work in healthy aircrew to improve their performance. ${ }^{[8,9]}$ Stroke volume calculations help assess improvement in stroke volume in real time during exercise. This along with Maximum oxygen consumption, Stroke volume improvement, Lactate threshold reached at level of exercise (in METS), VE/ CO2 slope and the usual exercise stress parameters based on ECG findings can be used to reflight aircrew with cardiopulmonary disorders in addition to other standard tests.

In our study, we observed that in our population a healthy aviator reaches an average of $80 \%$ of age predicted $\mathrm{VO} 2$ max [Table $1 \& 2$ 2] Normogram for age $30-39$ and $40-49$ attached above). Well trained and reasonably fit aviators despite having an infracted myocardial segment performed at more than $70 \%$ of $\mathrm{VO} 2$ max. Therefore, if an aviator performs upto $70 \%$ of age predicted $\mathrm{VO} 2$ max with anaerobic threshold being achieved at $40 \%$ of age predicted VO2 max he could be considered for reflghting. This data was used to safely reflight aviator no. 2,3,4,5 and 7 .

\section{Conclusion}

In this study, we conclude that in our population a healthy aviator reaches an average of $80 \%$ of age predicted VO2 max (normogram for age $30-39$ and $40-49$ attached above). Aviator no, 2,3,4,5,7 were reflighted, Therefore, if an aviator performs upto $70 \%$ of age predicted $\mathrm{VO} 2 \max$ 
with anaerobic threshold being achieved at $40 \%$ of age predicted VO2 max he could be considered for reflghting.

\section{References}

1. ATS/ACCP statement on cardiopulmonary exercise testing Am J Respir Care Med. 2003;167:211-277.

2. G. J. Balady, R. Arena, K. Sietsema, J. Myers, L. Coke, G. F. Fletcher, D. Forman, B. Franklin, M. Guazzi, M. Gulati, et al.Clinician's Guide to Cardiopulmonary Exercise Testing in Adults: A Scientific Statement From the American Heart Association Circulation, July 13, 2010; 122(2): $191-225$.

3. R. Gosselink, T. Troosters, D. Langer, and M. Decramer. Laboratory tests Clinical Exercise Testing, June 10, 2010; 129 - 147.

4. P. Palange, R. Antonucci, and G. Valli. Exercise testing in the prognostic evaluation of patients with lung and heart diseases. Clinical Exercise Testing, June 10, 2010; 195 - 207.

5. S. Glaser, C. Schaper, R. Ewert, and B. Koch. Peak oxygen uptake and exercise capacity: a reliable predictor of quality of life? Eur. Heart J.,
July 1, 2009; 30(13): $1674-1674$.

6. D. E. O'Donnell, D. Ofir, and P. Laveneziana. Patterns of cardiopulmonary response to exercise in lung diseases. Clinical Exercise Testing, June 10, 2010; 69 - 92.

7. M. Guazzi, K. Dickstein, M. Vicenzi, and R. Arena. Six-Minute Walk Test and Cardiopulmonary Exercise Testing in Patients With Chronic Heart Failure: A Comparative Analysis on Clinical and Prognostic Insights Circ Heart Fail, November 1, 2009; 2(6): 549 - 555.

8. R. Arena, J. Myers, M. A. Williams, M. Gulati, P. Kligfield, G. J. Balady, E. Collins, and G. Fletcher. Assessment of Functional Capacity in Clinical and Research Settings: A Scientific Statement From the American Heart Association Committee on Exercise, Rehabilitation, and Prevention of the Council on Clinical Cardiology and the Council on Cardiovascular Nursing. Circulation, July 17, 2007; 116(3): $329-343$.

9. R. Arena, J. Myers, and M. Guazzi. The Clinical Significance of Aerobic Exercise Testing and Prescription: From Apparently Healthy to Confirmed Cardiovascular Disease American Journal of Lifestyle Medicine, November 1, 2008; 2(6): 519 - 536.

Copyright: ( ) the author(s), 2019. It is an open-access article distributed under the terms of the Creative Commons Attribution License (CC BY 4.0), which permits authors to retain ownership of the copyright for their content, and allow anyone to download, reuse, reprint, modify, distribute and/or copy the content as long as the original authors and source are cited.

How to cite this article: Chauhan A, Marwaha MPS, Sharma V, Vaidya R, Anand BK. Cardiopulmonary Exercise Testing in Coronary Artery Disease Male Aviators Prior to Refighting: A Suggested Modality. Asian J. Med. Res. 2019;8(3):ME16-ME19.

DOI: dx.doi.org/10.21276/ajmr.2019.8.3.ME6 